\title{
A mixed boundary condition for accounting energy absorption and transmission in a diffusion equation model
}

\author{
Juan M. Navarro ${ }^{\text {a) }}$ \\ Grupo de Investigación en Telecomunicaciones Avanzadas (GRITA), Universidad Católica de Murcia \\ (UCAM), Murcia, 30107, Spain \\ José Escolano \\ Hudson Data, 25 Broadway, New York, New York 10004, USA
}

(Received 13 August 2018; revised 6 December 2018; accepted 10 December 2018; published online 30 April 2019)

\begin{abstract}
The acoustic diffusion equation model has shown to be a versatile alternative model for certain types of scenarios where the classical geometrical methods have been demonstrated to be inefficient or even inaccurate. In certain scenarios, the transmission loss plays a fundamental role for accounting, i.e., noise levels and sound propagation between rooms. This paper presents an extension of the absorption boundary conditions known as modified that unifies previously proposed boundary conditions in one equation, including transmission of energy between coupled rooms by both openings and enclosures. (C) 2019 Acoustical Society of America. https://doi.org/10.1121/1.5095883
\end{abstract}

$[\mathrm{NX}]$

Pages: $2718-2723$

\section{INTRODUCTION}

A diffusion equation for room acoustics modeling was proposed to model enclosures with low absorption and total diffuse reflection surfaces. ${ }^{1}$ During the last years, a considerable progress in this research line has been done, increasing the number of scenarios where the acoustic diffusion equation model can be applied. An important step forward has been the derivation of a diffusion equation model from the acoustic radiative transfer method, ${ }^{2}$ allowing a deeper understanding of the diffusion equation model and offering an interesting tool to work on extending the model for further applications. In Ref. 3, the acoustic radiative transfer method is used to extend the diffusion model to be applied with absorption boundary conditions, combining a proportion of specular and diffuse reflections. Moreover, another energetic wave equation model in room acoustics has been proposed from the propagation equation of radiative transfer. ${ }^{4}$

In this paper, a mixed boundary condition for accounting both energy absorption and transmission in a diffusion equation model is derived from the acoustic radiative transfer equation, resulting in an extension of the well-known modified boundary condition. ${ }^{5}$ This extension unifies previously proposed boundary conditions ${ }^{5-8}$ in one equation, including the effect of sound transmission through enclosures and openings. ${ }^{9,10}$

\section{ACOUSTIC DIFFUSION EQUATION MODEL}

The diffusion equation model for the sound energy density $w(\mathbf{r}, t)$ at position $\mathbf{r}$ and time $t$, defined on a domain $V$, with a sound source term $P(t)$ located at position $\mathbf{r}_{s}$, consisting of a partial differential equation with mixed boundary conditions $^{1,7}$ is fully described by

${ }^{\text {a)} E l e c t r o n i c ~ m a i l: ~ j m n a v a r r o @ u c a m . e d u ~}$

$$
\begin{aligned}
& \frac{\partial w(\mathbf{r}, t)}{\partial t}-D \nabla^{2} w(\mathbf{r}, t)=P(t) \delta\left(\mathbf{r}-\mathbf{r}_{s}\right) \text { in } V, \\
& -D \frac{\partial w(\mathbf{r}, t)}{\partial \mathbf{n}}=\frac{c \alpha(\mathbf{r})}{2[2-\alpha(\mathbf{r})]} w(\mathbf{r}, t) \text { on } \partial V,
\end{aligned}
$$

where $\nabla^{2}$ is the Laplace operator, and $D=4 V c / 3 S_{t}$ is the socalled diffusion coefficient where $c$ is the speed of sound, with volume $V$ and total interior area $S_{t}$.

Equation (2) is an absorption boundary condition that models the local effects on the sound field induced by different degrees of attenuation on surfaces. This boundary condition has different equations in technical literature. ${ }^{7}$ In this paper, the modified absorption factor is adopted to perform the simulations because it has shown the widest range of applicability, ${ }^{7}$ being $\alpha(\mathbf{r})$ the absorption coefficient of the surface at position $\mathbf{r}$.

With regard to the transmission loss modeling, Billon et al. ${ }^{8}$ proposed a modification of the diffusion model equation to account for sound transmission between two rooms. It consists of adding another boundary condition to the model to consider the room coupling through a partition wall with a term that represents the transmission of energy. Therefore, the following equation was enunciated:

$$
\begin{aligned}
D_{1} & \nabla w_{1}(\mathbf{r}, t) \cdot \hat{\mathbf{n}}+\frac{c \alpha(\mathbf{r})}{2[2-\alpha(\mathbf{r})]} w_{1}(\mathbf{r}, t) \\
= & \frac{c \tau w_{2}(\mathbf{r}, t)}{4} \text { on } S_{12},
\end{aligned}
$$

where $D_{1}$ is the diffusion coefficient of room $1, w_{1}$ and $w_{2}$ are the corresponding energy densities at both rooms, and $\tau$ is the transmission coefficient of the partition wall with the $S_{12}$ area. Another similar equation appears for taking into account the energy transfer from room 2 to room 1.

The main goal of this paper is to propose a new approach to extend the diffusion model equation with an 
expansion of the modified boundary condition ${ }^{5}$ that includes both transmission and absorption effects in a unified model. This model is formally derived from the acoustic radiative transfer equation and it allows simulating sound energy attenuation due to absorption of the surfaces together with sound energy propagation between different rooms of the same scenario.

\section{PROPOSED ENERGY ABSORPTION AND TRANSMISSION BOUNDARY CONDITION}

The diffusion equation model has been demonstrated to be an approach of the acoustic radiative transfer equation. When the sound radiance term is approached by using first order spherical harmonics, the diffusion equation model is obtained. ${ }^{2}$

To express the boundary conditions in the acoustic radiative transfer equation, the $S^{2}$ space, i.e., the unit sphere space, is partitioned into two hemispheres at each boundary point, designating the hemisphere where $(\hat{\mathbf{n}} \cdot \hat{\mathbf{s}})>0$ the positive hemisphere by $\Omega^{+}$, and the negative hemisphere by $\Omega^{-}$ analogously. The boundary conditions are also expressed in terms of the sound radiance $L(\mathbf{r}, \hat{\mathbf{s}}, t)$, which is defined as the energy flow at position $\mathbf{r}$ per unit normal area per unit solid angle per unit time $t$, where the normal area is perpendicular to the flow direction $\hat{\mathbf{s}}$. In the absence of sources at boundaries, see Fig. 1, the reflected sound radiance in a direction $\hat{\mathbf{s}}$ as a consequence of a sound particle traveling from direction $\hat{\mathbf{s}}^{\prime}$ and striking over a surface at position $\mathbf{r}_{b}$ is expressed as

$$
L\left(\mathbf{r}_{b}, \hat{\mathbf{s}}, t\right)=\int_{\Omega^{-}} r\left(\mathbf{r}_{b} ; \hat{\mathbf{s}}^{\prime}, \hat{\mathbf{s}}\right) L\left(\mathbf{r}_{b}, \hat{\mathbf{s}}^{\prime}, t\right)\left(\hat{\mathbf{s}}^{\prime} \cdot \hat{\mathbf{n}}\right) d \Omega^{\prime},
$$

where $r$ is the surface scattering or reflecting function with units of $\mathrm{sr}^{-1}$, defined as the probability that a particle at $\mathbf{r}_{b}$ moving in the $\hat{\mathbf{s}}^{\prime}$ direction will be reflected into a new direction $\hat{\mathbf{s}}$. The sound radiance leaving the surface is determined by solving the incoming sound radiation integral over the

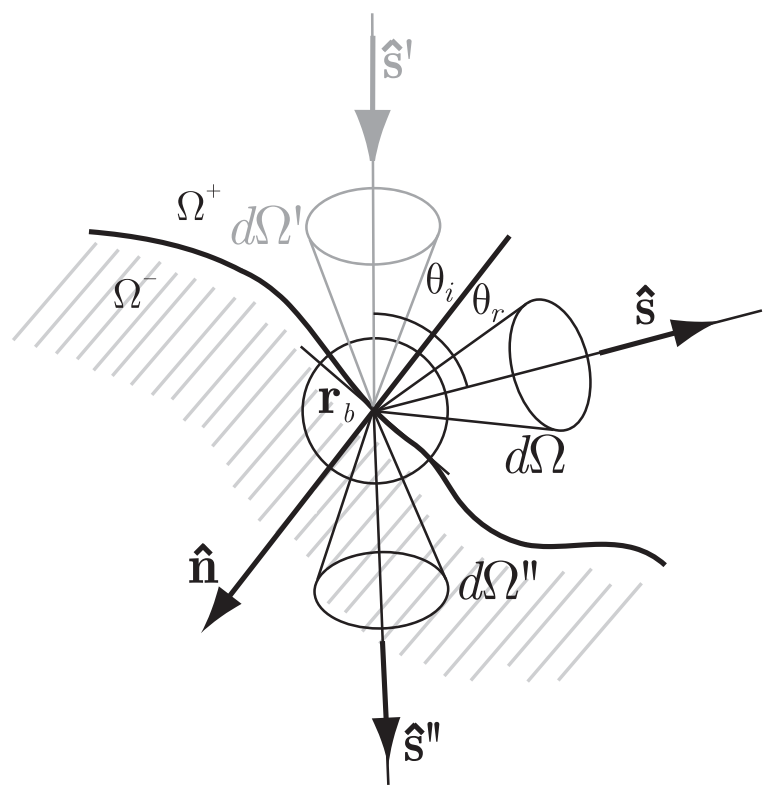

FIG. 1. The surface partitions with the set of directions $S^{3}$ at each point $\mathbf{r}_{\mathbf{b}} \cdot \hat{\mathbf{s}}^{\prime}$ is the incidence angle, $\hat{\mathbf{s}}$ is the reflection angle, and $\hat{\mathbf{s}}^{\prime \prime}$ is the transmission angle. positive hemisphere $\Omega^{+}$. When no transmission is considered, Eq. (2) can be mathematically approximated from Eq. (4), implicitly including the modified absorption factor. ${ }^{2}$

In order to expand these boundary conditions to include energy transmission and the consequent transmission losses, the following energy balance needs to be considered:

$$
r+\tau+\delta=1
$$

where $r$ is the reflection coefficient and $\delta$ accounts for the dissipation losses. The absorption coefficient is defined by $\alpha=\tau+\delta$ and therefore, $0 \leq \delta \leq \alpha$.

Then, following the scheme in Fig. 1, let us consider that a particle with sound radiance $L\left(\mathbf{r}, \hat{\mathbf{s}}^{\prime}, t\right)$ is traveling toward the direction $\hat{\mathbf{s}}^{\prime}$ and it reaches a boundary surface at point $\mathbf{r}_{b}$. Part of the radiance is reflected with a reflection factor $r$ in the direction $\hat{\mathbf{s}}$, whereas some other part of the radiance, with a proportion factor of $\tau$, passes through the boundary with direction $\hat{\mathbf{s}}^{\prime \prime}$. In terms of the acoustic radiative transfer model, it is expressed as follows:

$$
\begin{aligned}
\int_{\Omega^{+}} L\left(\mathbf{r}_{b}, \hat{\mathbf{s}}, t\right)(\hat{\mathbf{s}} \cdot \hat{\mathbf{n}}) d \Omega= & \int_{\Omega^{+}} r L\left(\mathbf{r}_{b}, \hat{\mathbf{s}}^{\prime}, t\right)\left(\hat{\mathbf{s}}^{\prime} \cdot \hat{\mathbf{n}}\right) d \Omega^{\prime} \\
& +\int_{\Omega^{-}} \tau L\left(\mathbf{r}_{b}, \hat{\mathbf{s}}^{\prime \prime}, t\right)\left(\hat{\mathbf{s}}^{\prime \prime} \cdot \hat{\mathbf{n}}\right) d \Omega^{\prime \prime},
\end{aligned}
$$

which denotes the energy conservation where the incident energy is divided into reflective and transmitted sound radiance. It should be noted that the dissipation losses have been considered to be negligible. Also, since the diffusion equation model assumes completely diffuse surfaces, the reflecting factor (and then implicitly, the absorption and transmission coefficients) is no longer directional dependent.

After integration, the diffusion approximation renders an expression for the sound radiation function (see Ref. 2 for details) and the boundary condition becomes

$$
\begin{aligned}
& \frac{w_{1}\left(\mathbf{r}_{b}^{+}, t\right)}{4}-\frac{\mathbf{J}_{1}\left(\mathbf{r}_{b}^{+}, t\right)}{2 c} \cdot \hat{\mathbf{n}} \\
& =\frac{\tau w_{2}\left(\mathbf{r}_{b}^{-}, t\right)}{4}-\frac{\tau \mathbf{J}_{2}\left(\mathbf{r}_{b}^{-}, t\right)}{2 c} \cdot \hat{\mathbf{n}}+\frac{r w_{1}\left(\mathbf{r}_{b}^{+}, t\right)}{4} \\
& \quad+\frac{r \mathbf{J}_{1}\left(\mathbf{r}_{b}^{+}, t\right)}{2 c} \cdot \hat{\mathbf{n}},
\end{aligned}
$$

where $w_{1}, \mathbf{J}_{1} \in \Omega^{+}$represents the energy density and energy flow at the boundary in the source or emitter room, while $w_{2}, \mathbf{J}_{2} \in \Omega^{-}$represents the adjacent or receiver room. The points $\mathbf{r}_{b}^{+} \in \Omega^{+}$and $\mathbf{r}_{b}^{-} \in \Omega^{-}$are situated over both sides of the boundary surface. It should be noted that despite the use of the subindex for labeling rooms may be redundant, it has been keep in order to be compared with Eq. (3).

By using the Fick's law $(\mathbf{J}=-D \nabla \omega)$ and expressing the reflection factor in terms of absorption coefficients $(r=1-\alpha)$, it results in

$$
\begin{aligned}
D_{1} & \nabla w_{1}\left(\mathbf{r}_{b}^{+}, t\right) \cdot \hat{\mathbf{n}}+\frac{c \alpha w_{1}\left(\mathbf{r}_{b}^{+}, t\right)}{2(2-\alpha)} \\
\quad= & \frac{c \tau w_{2}\left(\mathbf{r}_{b}^{-}, t\right)}{2(2-\alpha)}+\frac{\tau}{(2-\alpha)} D_{2} \nabla w_{2}\left(\mathbf{r}_{b}^{-}, t\right) \cdot \hat{\mathbf{n}} .
\end{aligned}
$$


This equation accounts for both energy absorption and transmission effects in a single equation. In order to validate the versatility of the model, let us present several extreme cases. If $\tau=0$, meaning that only scattering effects are considered with no transmission, Eq. (8) becomes the modified boundary condition [see Eq. (2)]. In the opposite case, when an opening is modelled, meaning that the entire energy is transmitted, e.g., $\tau=\alpha=1$, if an energy continuity is assumed, $w_{1}\left(\mathbf{r}_{b}^{+}, t\right)=w_{2}\left(\mathbf{r}_{b}^{+}, t\right)$, the equation becomes

$$
D_{1} \nabla w_{1}\left(\mathbf{r}_{b}^{+}, t\right) \cdot \hat{\mathbf{n}}-D_{2} \nabla w_{2}\left(\mathbf{r}_{b}^{-}, t\right) \cdot \hat{\mathbf{n}}=0,
$$

that corresponds to an opening boundary condition, ${ }^{12}$ indicating there is a flux discontinuity (or equivalently, energy flow continuity) condition that accounts for the effects of having a different diffusion coefficient in each room. The validation of flux discontinuity equation for the acoustic diffusion equation model can be found in Ref. 12 .

\section{SIMULATIONS AND RESULTS}

In order to validate the proposed model, several simulations and comparisons have been conducted for this purpose. To measure the transmission loss effect, the standardized level difference $D_{n T}$ is used as Ref. 13,

$$
D_{n T}=L_{1}-L_{2}+10 \log \left(T_{2} / T_{0}\right),
$$

where $L_{1}$ and $L_{2}$ are the sound pressure levels, expressed in $\mathrm{dB}$, measured in the primary/source and secondary/adjacent room, respectively, $T_{2}$ is the reverberation time of the secondary room and $T_{0}=0.5 \mathrm{~s}$ is a reference reverberation time. The insertion loss does not take into account the absorption conditions of the rooms. Therefore, the authors propose to use the $D_{n T}$ because it incorporates reverberation time dependency and it is being applied in international stand$\operatorname{ards}^{13}$ for room isolation measurements and predictions. It is demonstrated that if the total acoustic absorption area of the secondary room equals $10 \mathrm{~m}^{2}$, the parameter $D_{n T}$ can be theoretically estimated as,

$$
D_{n T}=R+10 \log \left(0.32 V_{2} / S_{S}\right)
$$

where $R$ is the sound reduction index or transmission loss that can be theoretically calculated as $-10 \log \tau, V_{2}$ is the volume of the secondary room, and $S_{S}$ is the surface of the wall partition.

An implementation of the diffusion equation model using a finite-difference time-domain model has been applied for the simulations. The Dufort-Frankel scheme has been chosen due to its unconditionally stable feature. In order to provide accurate results, the spatial sampling frequency in each direction has been set to $\Delta\{x, y, z\}=0.25 \mathrm{~m}$ and the sampling frequency $f_{s}$ has been set to $20 \mathrm{kHz}$, according to Ref. 11. The diffusion equation method models the time-dependent propagation of the sound energy density $\mathrm{w}(\mathbf{r}, \mathrm{t})$ in a room. Using an impulse function as input, the room impulse response is estimated. From the room impulse response, it is possible to calculate the sound pressure level and the reverberation time in both source and receiver rooms. ${ }^{14}$

First, the rooms are both proportional and equidimensional $(4 \times 4 \times 4 \mathrm{~m})$ to be in accordance with the statistical approach. The separating surface has dimensions $4 \times 4 \mathrm{~m}$. The norm EN-12354, ${ }^{13}$ with regard to the primary room, establishes that it needs to be highly reverberant; for that reason the absorption coefficient in the primary room is set to $\alpha_{1}=0.1$. The source and receiver are situated in the central point of their corresponding room.

In this test, the transmission loss $R$ varies from 0.25 to $30 \mathrm{~dB}$. Figure 2(a) plots the standardized level difference by using the statistical theory [Eq. (11)], the proposed model [Eq. (8)], and Billon's approach [Eq. (3)]. The depicted graphs in Fig. 2(a) show how both models show similar behaviour to the statistical model for those values above $5 \mathrm{~dB}$. However, it can be observed that there is an observable difference between both numerical approaches due principally to the boundary conditions used in each model. Although this difference is not very significant, it is expected when using the modified boundary conditions, as stated in Ref. 7.

An additional analysis has been conducted by altering the secondary room dimensions to have $20 \mathrm{~m}$ on the $y$ axis [see Fig. 2(b)]. Despite the fact the results from both simulation models present small differences as mentioned above, for the scenario depicted in Fig. 2(b), the difference between both simulation models and the statistical theory increases when increasing volume. However, it has to be noticed that this difference is mostly caused by the fact that one of the dimensions is significantly larger than the rest, becoming a so-called disproportionate room. It has been demonstrated that the statistical theory does not apply to this scenario whereas the diffusion equation model does. ${ }^{15}$ The differences of the proposed model when compared to Billon's approach are mainly noticiable when $\tau$ tends to 1 and when the volume of the secondary room becomes comparatively bigger than the primary room. Again, these differences are in accordance with the differences between the use of a modified boundary condition and Sabine's formula. Therefore, the proposed model allows to incorporate both the modified boundary condition and a transmission coefficient in an unified manner, adding accuracy to the model for those scenarios where the modified boundary condition provides significant advantages, as reported by Ref. 7 .

Another interesting scenario is to compare results from both diffusion-based simulation methods when the coupling surface has some opening area, which is the extreme case when $\tau=1$, and the rest is a closed area with absorption and transmission losses. To test this situation, a second batch of simulations has been carried out using the coupled-volume system scenario in Ref. 12. In that paper, a model scaled coupled volume is used to compare with the performance of the diffusion equation model. The scope of that work was to validate the diffusion equation model to predict the double slope decay by varying the aperture width.

To model an aperture in a diffusion equation model, a flux discontinuity condition is used, the same as that 

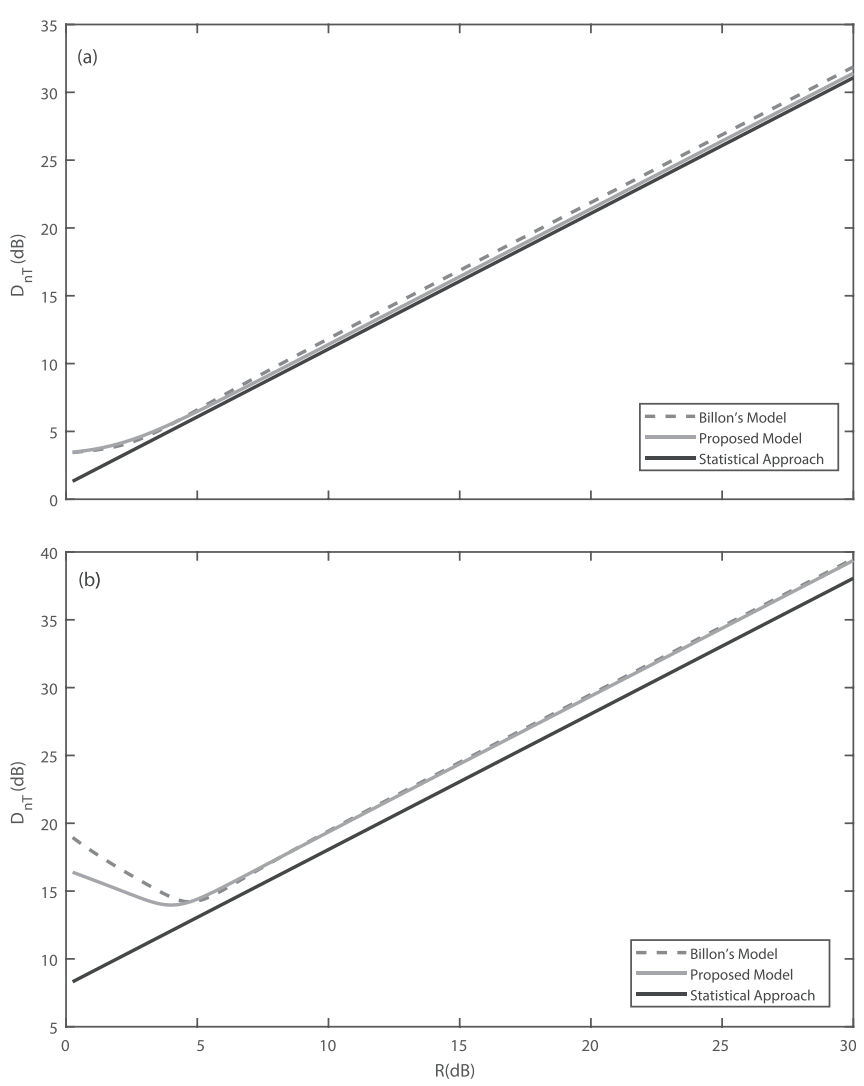

FIG. 2. The standardized level difference calculated by using the statistical theory, the proposed model, and Billon's approach where $R$ varies from 0.25 to $30 \mathrm{~dB}$ and secondary room dimensions are (a) $4 \times 4 \times 4 \mathrm{~m}$ and (b) $4 \times 20 \times 4 \mathrm{~m}$.

obtained in Eq. (9). The coupled volume scenario consists of two rooms with highly diffuse walls and which dimensions, absorption, and natural reverberation times are summarized at Table I. In this particular case, two aperture widths have been arranged of 40 and $60 \mathrm{~cm}$, having in both cases a height of $7.20 \mathrm{~m}$, the same as the rooms. Sound source finds itself in the lower-left corner of the primary room, the farthest away from the coupling aperture. For the receiver positions, a grid of three rows and ten columns is defined toward the upper left corner of the primary room. More details about the model setup and the finite difference implementation can be found in Ref. 12.
TABLE I. Dimensions, absorption, and natural reverberation times of the coupled rooms model in Ref. 12.

\begin{tabular}{lcc}
\hline \hline Feature & Primary Room & Secondary Room \\
\hline Dimensions & $4.88 \times 6.32 \times 7.20 \mathrm{~m}$ & $7.60 \times 9.76 \times 7.20 \mathrm{~m}$ \\
Volume & $222.06 \mathrm{~m}^{3}$ & $534.07 \mathrm{~m}^{3}$ \\
Absorption & 0.41 & 0.22 \\
Natural RT & $0.47 \mathrm{~s}$ & $1.17 \mathrm{~s}$ \\
\hline \hline
\end{tabular}

Both diffusion-based simulation methods have been implemented in the same model. In order to check if the proposed boundary conditions meet the flow continuity, Fig. 3 plots the spatial variation of sound pressure level normalized along the $x$ axis (length) in two lines; one crossing the aperture $(y=6.1 \mathrm{~m}$ and $z=3.6 \mathrm{~m})$ and one crossing the wall $(y=3.1 \mathrm{~m}$ and $z=3.6 \mathrm{~m})$. It is observed in the solid curve how the energy density gradually continues when the opening is crossed. However, a discontinuity in the energy density is shown in the dashed curve due to the losses in the wall as expected. ${ }^{16}$,

In Fig. 4, sound level energy decay responses of the receiver situated at row 2 of column 1 for aperture width of 40 and $60 \mathrm{~cm}$ are shown comparing both models. Using the energy decay profiles of both approaches, the parameters of these temporal decays, reverberation times of both room $T_{1}$ and $T_{2}$, level differences $\Delta L$, and turning point time can be estimated. From the comparison of both numerical models with respect to the measurement calculated parameters, see Table II, several conclusions can be drawn. Regarding reverberation times, no significant differences are observed between both models and the measurements. Moreover, both approaches are significantly accurate and no observable differences exist between both models. However, in terms of $\Delta L$ and turning point time, there exists a remarkable difference between both numerical models and the measurements, where the proposed model is discernibly more accurate, whereas Billon's approach tends to noticeably overestimate both variables, with a bigger difference between experimental and estimated values. From the results, it is evidenced that there is a strong resemblance in terms of decay times; however, there is a significant difference in terms of level difference and turning point, ${ }^{17}$ therefore the proposed model provides better estimations for couple volumes analysis.

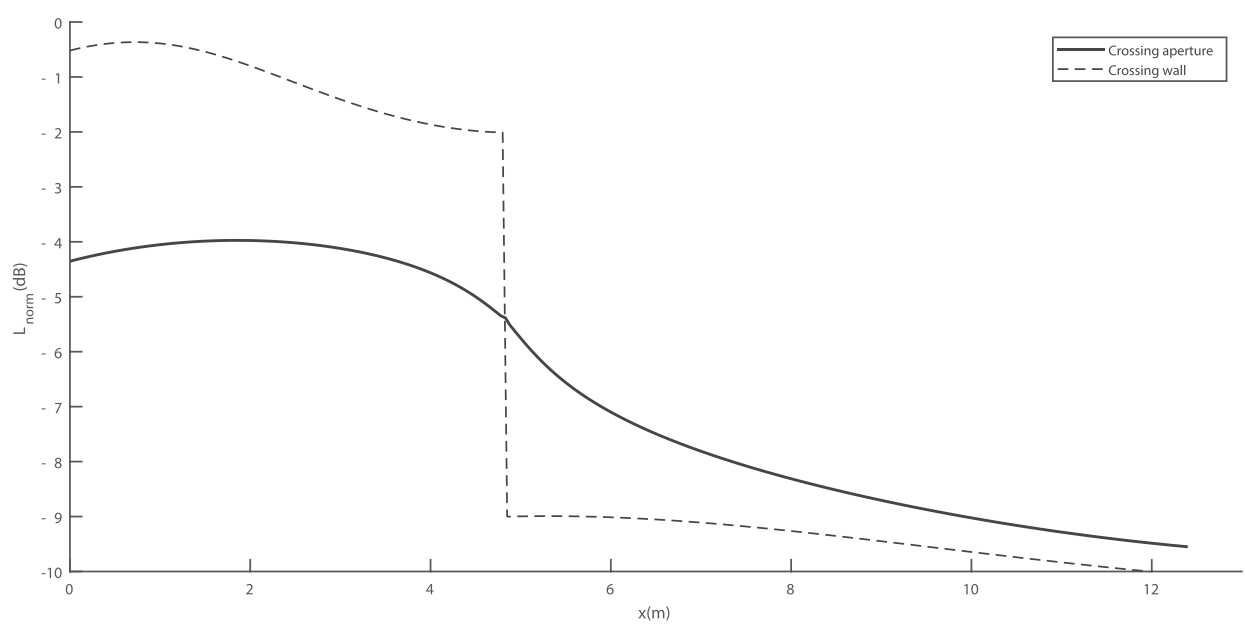

FIG. 3. Spatial variation of sound pressure level normalized calculated with the proposed model along the $x$ axis (length) in two lines; one crossing the aperture and one crossing the wall. 


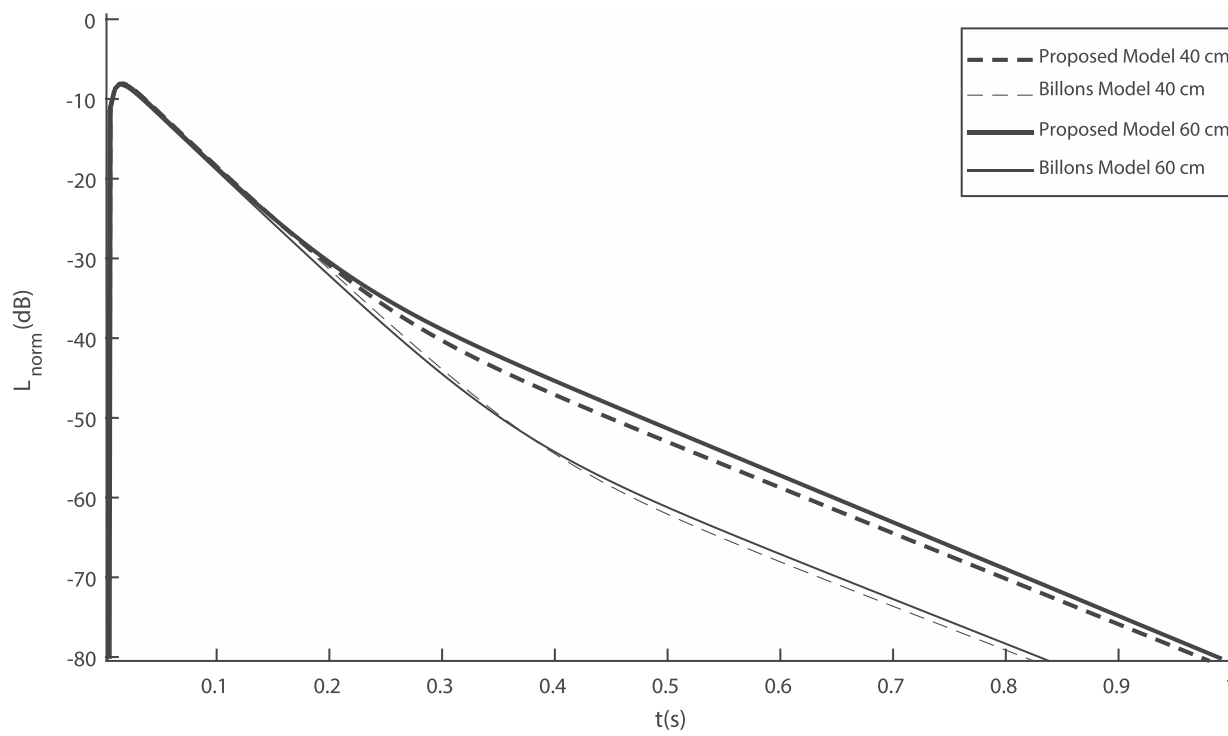

FIG. 4. Sound pressure level normalized from decays over time at receiver row 2 column 1 location at the proposed model and Billon's model simulations for aperture widths of 40 and $60 \mathrm{~cm}$.

\section{CONCLUSIONS}

In this work a mixed boundary condition for the diffusion equation model for accounting energy transmission between rooms has been presented. This new boundary condition allows integrating both the absorption coefficients and the transmission loss properties of the materials in a single expression and it extends the application of the diffusion model to complex scenarios. Moreover, this proposed model takes advantage of the modified boundary condition, as a more accurate alternative to Sabine or Eyring's formulas. At the same time, it incorporates the effects due to energy transmission across openings and enclosures between different domains or volumes.

The proposed boundary condition for sound energy transmission has been derived using the radiative transfer approach. Sound radiance allows to define, assuming completely diffuse reflection, a new boundary condition that includes reflection, absorption, and transmission of sound energy.

Several simulations have been carried out to support the proposed model. In the first scenario with coupled rooms by a wall, results have been compared with previous Billon's boundary conditions approach and statistical theory. Simulations have shown great agreement of both boundary models with statistical theory when the dimensions of the receiver room are proportionate. A negligible difference has appeared for low transmission coefficients that is more pronounced for a disproportionate room-shape.

TABLE II. Decay parameters from experimental data in Ref. 12 and calculated from diffusion-equation model predicted decay functions shown in Fig. 4.

\begin{tabular}{lccc}
\hline \hline Boundary model & Scale Model & Proposed Model & Billon's Model \\
\hline Aperture width $(\mathrm{cm})$ & $40 / 60$ & $40 / 60$ & $40 / 60$ \\
$T_{1}(\mathrm{~s})$ & $0.45 / 0.45$ & $0.44 / 0.42$ & $0.44 / 0.42$ \\
$T_{2}(\mathrm{~s})$ & $1.51 / 1.03$ & $1.48 / 1.02$ & $1.35 / 1.02$ \\
$\Delta L(\mathrm{~dB})$ & $20 / 18$ & $17 / 14$ & $28 / 24$ \\
Turning point time $(\mathrm{ms})$ & $326 / 290$ & $280 / 220$ & $410 / 350$ \\
\hline \hline
\end{tabular}

The second set of simulations proposed a complex scenario with coupled rooms by an aperture and an enclosure. Spatial variation of energy density across the aperture and across the wall gave validation to the energy continuity and discontinuity, respectively, in the proposed boundary condition. Moreover, temporal energy room responses show that there is a strong resemblance with experimental data in terms of decay times between both boundary models. However, the proposed model has provided a better estimation in terms of level difference and turning point compared to experimental data.

\section{ACKNOWLEDGMENTS}

This work has been supported by the Spanish Ministry of Economy and Competitiveness under Project No. TIN2016-78799-P.

${ }^{1}$ V. Valeau, J. Picaut, and M. Hodgson, "On the use of a diffusion equation for room-acoustic prediction," J. Acoust. Soc. Am. 119, 1504-1513 (2006).

${ }^{2}$ J. M. Navarro, F. Jacobsen, J. Escolano, and J. J. López, “A theoretical approach to room acoustic simulations based on a radiative transfer model," Acta Acust. Acust. 96, 1078-1089 (2010).

${ }^{3}$ C. Foy, J. Picaut, and V. Valeau, "Including scattering within the room acoustics diffusion model: An analytical approach," J. Acoust. Soc. Am. 140, 2659-2669 (2016).

${ }^{4}$ J. D. Polack, H. Dujourdy, B. Pialot, and T. Toulemonde, "Energetic wave equation for diffuse sound fields," J. Acoust. Soc. Am. 142, 2560-2560 (2017).

${ }^{5}$ Y. Jing and N. Xiang, "A modified diffusion equation for room-acoustic predication,” J. Acoust. Soc. Am. 121, 3284-3287 (2007).

${ }^{6}$ A. Billon, J. Picaut, and A. Sakout, "Prediction of the reverberation time in high absorbent room using a modified-diffusion model," Appl. Acoust. 69, 68-74 (2008).

${ }^{7}$ Y. Jing and N. Xiang, "On boundary conditions for the diffusion equation in room acoustic prediction: Theory, simulations, and experiments," J. Acoust. Soc. Am. 123, 145-153 (2008).

${ }^{8}$ A. Billon, C. Foy, J. Picaut, V. Valeau, and A. Sakout, "Modeling the sound transmission between rooms coupled through partition walls by using a diffusion model," J. Acoust. Soc. Am. 123(6), 4261-4271 (2008).

${ }^{9}$ A. Billon, V. Valeau, A. Sakout, and J. Picaut, "On the use of a diffusion model for acoustically coupled rooms," J. Acoust. Soc. Am. 120, 2043-2054 (2006). 
${ }^{10}$ N. Xiang, Y. Jing, and A. Bockman, "Investigation of acoustically coupled enclosures using a diffusion-equation model," J. Acoust. Soc. Am. 126, 1187-1198 (2009).

${ }^{11}$ J. M. Navarro, J. Escolano, and J. J. López, "Implementation and evaluation of a diffusion equation model based on finite difference schemes for sound field prediction in rooms," Appl. Acoust. 73, 659-665 (2012).

${ }^{12}$ N. Xiang, J. Escolano, J. M. Navarro, and Y. Jing, "Investigation on the effect of aperture sizes and receiver positions in coupled rooms," J. Acoust. Soc. Am. 133(6), 3975-3985 (2013).

${ }^{13}$ EN 12354-1:2000. Building Acoustics. Estimation of acoustic performance of buildings from the performance of elements. Part 1: Airborne sound insulation between rooms (2000).
${ }^{14}$ J. M. Navarro and J. Escolano, "Simulation of building indoor acoustics using an acoustic diffusion equation model," J. Bldg. Perform. Simul. 8(1), 3-14 (2015).

${ }^{15}$ J. Picaut, L. Simon, and J.-D. Polack, "Sound field in long rooms with diffusely reflecting boundaries," Appl. Acoust. 56, 217-240 (1999).

${ }^{16}$ N. Xiang, P. Goggans, T. Jasa, and P. Robinson, "Bayesian characterization of multiple-slope sound energy decays in coupled-volume systems," J. Acoust. Soc. Am. 129, 741-752 (2011).

${ }^{17}$ M. Meissner, "Acoustic energy density distribution and sound intensity vector field inside coupled spaces," J. Acoust. Soc. Am. 132, 228-238 (2012). 